\title{
Characteristics of Second Primary Breast Cancer after Ovarian Cancer: A Korea Central Cancer Registry retrospective study
}

\section{Eun-Gyeong Lee}

National Cancer Center

Jiwon Lim

National Cancer Control Institute, National Cancer Center

Hyeong In $\mathrm{Ha}$

Pusan National University Yangsan Hospital, Pusan National University School of Medicine

Myong Cheol Lim

National Cancer Center

Yoon Jung Chang

National Cancer Control Institute, National Cancer Center

\section{Young-Joo Won}

National Cancer Control Institute, National Cancer Center

So-Youn Jung ( $\nabla$ goje1@ncc.re.kr)

National Cancer Center

\section{Research Article}

Keywords: ovarian cancer, breast cancer, second primary, survival, incidence

Posted Date: October 5th, 2021

DOl: https://doi.org/10.21203/rs.3.rs-900227/v1

License: (9) This work is licensed under a Creative Commons Attribution 4.0 International License. Read Full License 


\section{Abstract}

Background: Second primary cancer has become an important issue among cancer survivors. This study sought to determine the differences in clinicopathologic outcomes between second primary breast cancer (SPBC) after ovarian cancer and primary breast cancer (PBC) in Korea.

Methods: We searched the Korea Central Cancer Registry and identified 251,244 breast cancer cases that were diagnosed between 1999 and 2017. Demographic and clinical characteristics and overall survival (OS) rates were estimated according to age, histological type, and cancer stage.

Results: Among the 228,329 patients included, 228,148 were patients with PBC and 181 were had SPBC, diagnosed after ovarian cancer (OC). The most common ages at diagnosis were 50-59 years for SPBC and 40-49 years for PBC. Patients with SPBC were significantly less likely than patients with PBC to receive adjuvant radiotherapy $(14.92 \%$ vs. $21.92 \%, p=0.0228)$ or adjuvant chemotherapy $(44.75 \%$ vs. $55.69 \%, p=0.0031$ ). Based on the age-standardized rate (ASR), the incidence of SPBC after OC was 293.58 per 100,000 ovarian patients and the incidence of PBC was 39.13 per 100,000 women. The 5-year OS rates were $72.88 \%$ and $89.37 \%$ for SPBC and PBC $(p<0.0001)$. The OS rate in SPBC decreased significantly with advanced stage and older age.

Conclusion: The incidence of breast cancer is about 1.27 times higher in ovarian cancer patients than in healthy people. The survival outcomes were worse for SPBC than for PBC and were related to older age and advanced stage. Active screening for breast cancer is necessary in ovarian cancer patients.

\section{Background}

Breast cancer is the most common female cancer and it is steadily increasing worldwide. [1] On the other hand, ovarian cancer has the highest mortality rate among gynecologic malignancies. [2] Korea has followed the global trend in this respect. According to 2016 nationwide cancer statistics in Korea, breast cancer had the highest incidence in women, with a crude incidence rate of 85.0 per 100,000; additionally, about 2,600 people were newly diagnosed with ovarian cancer, which caused an estimated 1,200 deaths in 2016. [3]

Survivors of primary cancer are increasing with advances in diagnostic methods and treatments. Consequently, second primary cancer has become an important issue among survivors. Various studies on second primary breast cancer following ovarian cancer have reported that this cancer is related to genetic factors, such as BRCA mutations. [4-6] For these patients, the risk of breast cancer was reduced by treatment of ovarian cancer, which consists of salpingo-oophorectomy and platinum-based chemotherapy. [7, 8] Some studies have reported differences in the clinical and survival outcomes and the risk of second primary breast cancer. [9-11] However, these have mainly originated from western countries, and reports from Asian countries have been limited. 
This study aimed to determine the risk and survival outcomes for patients with second primary breast cancer (SPBC) diagnosed after ovarian cancer. We also aimed to identify differences in clinicopathologic outcomes between second primary breast cancer and primary breast cancer (PBC).

\section{Methods}

\section{Study population}

Using the population-based Korea Central Cancer Registry (KCCR) database, we searched all patients with breast cancer who were diagnosed with PBC and SPBC after ovarian cancer between 1999 and 2017. We excluded breast cancer with other secondary cancers from the analysis. The KCCR was established in 1999 and is a representative population registry that collects about $98 \%$ of cancer data in Korea.

The different types of breast cancer (C50) and ovarian cancer included the following: primary peritoneal cancer (C48), epithelial ovarian cancer (C56.9), and fallopian tube cancer (C57) which were all defined based on the basis of the International Classification of Diseases for Oncology, 3rd edition (ICD-0-3). [12]

Patients were grouped according to age ( $<30$ years, $30-39$ years, $40-49$ years, $50-59$ years, $60-69$ years, 70-79 years, and $\geq 80$ years), date of diagnosis; Surveillance, Epidemiology, and End Results (SEER) stage; and the course of treatment, such as operation, radiation therapy, and chemotherapy within 4 months after the diagnosis of breast cancer.

\section{Statistical analysis}

The age-standardized rate (ASR) was calculated in patients diagnosed with breast cancer, using Segi's world standard population and was expressed per 100,000 people. The standardized incidence ratio (SIR) of subsequent breast cancer among patients with ovarian cancer was calculated to quantify relative risk compared to the general population. Survival curves were created using the Kaplan-Meier method and compared using the log-rank test, according to age, histological type, and SEER stage. All statistical tests were considered statistically significant at $p$-values of $<0.05$. All statistical analyses were performed using SAS software (version 9.4; SAS Institute, Inc., Cary, NC, USA), SEER* Stat (version 8.3.6; National Cancer Institute, Bethesda, MD, USA), and STATA software (version 16; StataCorp LLC, College Station, TX, USA).

\section{Results}

\section{Characteristics of second primary breast cancer after ovarian cancer}

According to the KCCR database, 251,244 patients were newly diagnosed with breast cancer from 1999 to 2017 , of whom 228,148 were PBC patients and 181 were patients with SPBC diagnosed with ovarian 
cancer. As shown in Table 1, the ASR for breast cancer was 39.1288 per 100,000 women. The ASR of SPBC was 0.0280 per 100,000 women and 293.58 per 100,000 after ovarian patients.

\section{Table 1}

Age-Standardized Rate of breast cancer by age, 1999-2017

\begin{tabular}{|c|c|c|c|c|c|c|c|}
\hline \multirow[t]{2}{*}{ Age(yrs) } & \multicolumn{3}{|c|}{ Breast cancer } & \multicolumn{4}{|c|}{$\begin{array}{l}\text { Second primary breast cancer } \\
\text { after ovarian cancer }\end{array}$} \\
\hline & $\begin{array}{l}\text { ASR* } \\
\text { per } \\
100,000 \\
\text { women }\end{array}$ & $\begin{array}{l}\% \text { of } \\
\text { cases }\end{array}$ & Cases & $\begin{array}{l}\text { ASR* } \\
\text { per } \\
100,000 \\
\text { women }\end{array}$ & $\begin{array}{l}\text { ASR } \\
\text { per } 100,000 \\
\text { ovarian cancer } \\
\text { patients }\end{array}$ & $\begin{array}{l}\% \text { of } \\
\text { cases }\end{array}$ & Cases \\
\hline$<30$ & 1.0873 & 1.36 & 3418 & 0.0003 & 44.13 & 0.55 & 1 \\
\hline $30-39$ & 4.9351 & 12.81 & 32181 & 0.0015 & 44.61 & 5.52 & 10 \\
\hline $40-49$ & 14.2082 & 36.41 & 91490 & 0.0068 & 69.80 & 24.31 & 44 \\
\hline $50-59$ & 10.6854 & 27.76 & 69736 & 0.0090 & 60.80 & 32.60 & 59 \\
\hline $60-69$ & 6.1176 & 13.96 & 35069 & 0.0080 & 53.69 & 25.41 & 46 \\
\hline $70-79$ & 1.7397 & 6.06 & 15216 & 0.0019 & 15.09 & 9.39 & 17 \\
\hline$\geq 80$ & 0.3556 & 1.65 & 4134 & 0.0003 & 5.46 & 2.21 & 4 \\
\hline Total & 39.1288 & 100.00 & 251244 & 0.0280 & 293.58 & 100.00 & 181 \\
\hline
\end{tabular}

* ASR (Age-Standardized Rate) was calculated using Segi's world standard population; yrs, years

Table 2 shows the clinicopathological characteristics of patients with breast cancer. The mean age at diagnosis of patients with SPBC after ovarian cancer was $56.09 \pm 10.81$ years and that of patients with PBC was $50.65 \pm 11.40$ years. The SPBC was diagnosed at a significantly older age than the PBC ( $p<$ $0.0001)$. The most common age group at SPBC diagnosis was 50-59 years $(32.60 \%)$, while the most common age group for diagnosis of PBC was $40-49$ years $(37.07 \%, p<0.0001)$. Both groups were most frequently diagnosed with local stage $(65.71 \%$ of SPBC vs. $55.70 \%$ of PBC cases, $p=0.1844)$ and the invasive carcinoma of no special type (NST) was the most common histological type (84.53\% of SPBC vs. $88.98 \%$ of PBC cases, $p=0.0072$ ). Surgery was performed in $82.87 \%$ of SPBC cases and $86.94 \%$ of $\mathrm{PBC}$ cases $(p=0.105)$.

Table 2

Clinicopathologic characteristics of patients with breast cancer in Korea, 1999-2017 
Characteristic

SIRq Second primary breast cancer after ovarian cancer

Cases (Total patients)

$1.27 * \quad 181(178)$

$8.95(4.87)$

primary

breast

Pcancer

Follow-up (yr.): Mean (SD)

$\begin{array}{lll}8.95(4.87) & 6.38 & <0.0001\end{array}$

Follow-up from second primary breast cancer diagnosis (yr): Mean (SD)

$3.93(3.74)$

Age at breast cancer diagnosis: Mean $\quad$ - $\quad 56.09$ (10.81)

50.65

$<0.0001$ (SD)

Age at diagnosis of ovarian cancer (yr.): Mean (SD)

$51.17(10.14)$

Age group at ovarian cancer diagnosis

(yr.)

\begin{tabular}{llll}
$<30$ & 3.71 & $1(0.55 \%)$ & $\begin{array}{l}3235 \\
(1.42 \%)\end{array}$ \\
\hline $30-39$ & 1.73 & $10(5.52 \%)$ & $\begin{array}{l}30306 \\
(13.28 \%)\end{array}$ \\
\hline $40-49$ & 1.12 & $44(24.31 \%)$ & $\begin{array}{l}84582 \\
(37.07 \%)\end{array}$ \\
\hline $50-59$ & 1.06 & $59(32.60 \%)$ & $\begin{array}{l}63006 \\
(27.62 \%)\end{array}$ \\
\hline $60-69$ & $1.53^{*}$ & $46(25.41 \%)$ & $\begin{array}{l}30595 \\
(13.41 \%)\end{array}$ \\
\hline $70-79$ & 1.60 & $17(9.39 \%)$ & $\begin{array}{l}13126 \\
(5.75 \%)\end{array}$ \\
\hline 80 & 2.84 & $4(2.21 \%)$ & $\begin{array}{l}3298 \\
(1.45 \%)\end{array}$ \\
\hline
\end{tabular}

Histologic type

$\mathrm{NST}^{\mathrm{c}}$

$1.21^{*} 153(84.53 \%)$

203006

(88.98\%)

0.0072

Lobular

$0.86 \quad 5(2.79 \%)$

8334

$(3.65 \%)$

Mucinous

$0.71 \quad 2(1.1 \%)$

4353

$(1.91 \%)$

Medullary

$3.583(1.66 \%)$

1894

$(0.83 \%)$

Metaplastic

$0.97 \quad 1(0.55 \%)$

1424

$(0.62 \%)$ 
Papillary

$4.36 * \quad 6(3.31 \%)$

Others

$2.53 * \quad 11(6.08 \%)$

Stage at diagnosis (Since 2006)

Local

Regional

Distant

Unknown

Radiation therapy

$0.92 \quad 27(14.92 \%)$

$1.35^{\star} \quad 154(85.08 \%)$
99481

$(55.70 \%)$

0.1844

60777

(34.03\%)

\section{4}

$(4.86 \%)$

9675

$(5.42 \%)$
Yes

No

Yes

No

\section{Surgery}

Chemotherapy

Yes

No
198342

$(86.94 \%)$

$1.53^{*} 31(17.13 \%)$

29806

$(13.06 \%)$
0.1050

50010

(21.92\%)

0.0228

178138

(78.08\%)

Interval between 1 st and 2 nd cancers

(yr.)

\begin{tabular}{lll} 
Mean (SD) & - & 5.05 (3.98) \\
$<1$ & 1.05 & 21 \\
$1-4$ & $1.34^{\star}$ & 86 \\
\hline $5-9$ & 1.31 & 50 \\
$\geq 10$ & 1.16 & 24
\end{tabular}

ISIR: Standardized Incidence Ratio ${ }^{\star \star}$ ANOVA tests and chi-square tests were performed to evaluate 
differences by factor for continuous variables, and for categorical variables, respectively. ${ }^{\mathrm{c}}$ NST: No Special Type

* Significant at alpha $=0.5$

The SIR for SPBC was 1.27 in the patients overall, and 1.53 in women aged 60-69 years. The papillary type showed a significantly higher SIR (4.36). The SIR of the NST subtype was 1.21 . When comparing the SIR according to surgical status, the SIR was significantly higher when surgery was not performed (SIR 1.22 with surgery vs. SIR 1.53 without surgery). In terms of additional treatment, the SIR was 1.35 without radiation therapy, and 1.47 without chemotherapy.

A total of 181 women diagnosed with SPBC were evaluated for a mean follow-up period of 8.95 years. The mean interval from the diagnosis of ovarian cancer to the diagnosis of SPBC was 5.05 years. In this group, the SIR between 1 and 4 years increased significantly to 1.34 .

\section{Survival outcomes}

The 5-year OS rate from the onset of SPBC was $72.88 \%$ and that from the onset of PBC was $89.37 \%$ (Figure 1). Compared by age, the 5 -year OS rate of patients with SPBC was low, at $50.05 \%$, in patients aged 70-79 years and the median survival time was 61 months (Figure 2). The 5-year OS rate of patients aged $40-49$ years was also low, at $67.95 \%$, and the median survival time was 135 months. For PBC, the 5 -year OS rate was $44.43 \%$ in patients over 80 years of age, and the median survival time was 51 months. The 5 -year OS rate was $77.21 \%$ in patients aged $70-79$ years and the medial survival time was 151 months.

Among cases with NST histology, the 5-year OS rates were $72.07 \%$ in SPBC and $89.16 \%$ in PBC cases. When compared by stage, patients with SPBC had a significantly worse prognosis than those with PBC.

\section{Discussion}

In this study, we investigated differences in clinicopathologic outcomes between SPBC after ovarian cancer and PBC in Korea. We showed that the incidence of breast cancer is higher in patients with ovarian cancer than it is in healthy people and that survival outcomes were worse for SPBC than for PBC; increased mortality was related to older age and advanced cancer stage.

In the current study, SPBC after ovarian cancer accounted for only $0.07 \%$ of newly diagnosed breast cancers in Korea, based on KCCR data from a 19-year period. In a retrospective observational study using SEER data between 1973 and 2013, the incidence of SPBC in the ovarian cancer cohort was approximately $1.37 \%(1,821 / 133,149)$ and the incidence of SPBC decreased from $7.2 \%$ to $2.0 \%$ between 1973 and $2008(P<0.05)$. [13] From 1999 to 2017, the incidence of breast cancer continued to increase year by year. On the other hand, the incidence of SPBC after ovarian cancer did not remain constant. In 
this study, the incidence of SPBC after ovarian cancer increased about two-fold in 2016 and 2017 (Supplement 1). Increasing rates of SPBC may be due to interest in genetic testing and application of insurance reimbursement, which may have led to active screening for breast cancer.

Ovarian cancer and breast cancer are hereditary cancers, and BRCA1 and BRCA2 are well known as causative genes. [4] According to the National Comprehensive Cancer Network guideline, risk-reducing surgery is recommended for carriers of $B R C A$ gene mutations at an appropriate time. [7] A previous metaanalysis reported that the risk of breast cancer was reduced by about $50 \%$ in carriers of $B R C A 1$ and BRCA2 mutations who underwent risk-reducing bilateral salpingo-oophorectomy (RRSO). [8] In our previous study about patterns of risk-reducing surgery in carriers of $B R C A$ mutations, the rate of RRSO was $42.5 \%$. After insurance reimbursement started in 2013, the rate of risk-reducing surgery was significantly higher (46.3\% after 2013 vs. $31.6 \%$ before $2013, p<0.001)$. [14]

In the current study, the overall SIR for SPBC after ovarian cancer was $1.27(95 \% \mathrm{Cl}, 1.09-1.46)$. In a study of invasive ovarian cancer, the SIR of breast cancer was 1.35 ( $95 \% \mathrm{Cl}, 0.85-2.05)$. [10] In another study on an ovarian cancer cohort from the Stockholm-Gotland Cancer Registry, the SIR of breast cancer was $1.41(95 \% \mathrm{Cl}, 1.14-1.75)$. [9] We found that the SIR of SPBCS was significantly higher at 1.34 between 1 and 4 years after the initial diagnosis of ovarian cancer. Thus, patients with ovarian cancer require more active surveillance for SPBC during this period.

This study showed that patients with SPBC after ovarian cancer had a markedly poor survival. Interestingly, when compared by age, our study confirmed that the survival rate of patients with PBC was relatively lower in the older age-group, whereas the survival rate of patients with SPBC was lower in the 40s age-group. Treatment of ovarian cancer with radiation therapy and chemotherapy may lead to aggressiveness of the second primary tumor. A previous study using the SEER program database showed that survival of patients with localized SPBC was similar to that of patients with PBC, while cases with advanced stage SPBC showed worse survival outcomes. [11] Our study showed similar results.

This study had several strengths. We analyzed a population-based cancer registry that included approximately $98 \%$ of Korean cancer cases. No previous study has reported the clinical outcomes of patients with breast cancer after ovarian cancer in Korea. However, our study had several limitations. First, this study lacked detailed clinical information regarding surgery type, such as mastectomy or breast-conserving surgery, and subtypes of tumor, such as luminal A, luminal B, HER2-positive, and triplenegative. Second, the study did not have access to personal history, family history and status of genetic mutations, such as those in $B R C A 1 / B R C A 2$, which influence hereditary cancer. Further studies should perform detailed investigations regarding the breast cancer characteristics.

\section{Conclusion}

The present study confirmed that the incidence of SPBC in patients with ovarian cancer was higher than that observed in those with primary breast cancer, and the survival outcome was low. However, if it the cancer is detected in the early stages, it shows a survival rate similar to those with PBC. Hence, active 
screening for breast cancer is necessary in patients with ovarian cancer between 1 and 4 years after diagnosis of ovarian cancer.

\section{Declarations}

\section{Acknowledgements}

This work was supported by the National Cancer Center Grant (NCC-1910132, NCC-1911272, NCC1911274).

\section{Availability of data and materials}

All data generated or analysed during this study are included in this published article [and its supplementary information files].

\section{Ethics approval and consent to participate}

This article does not contain animals performed by any of the authors. The requirement for obtaining informed patient consent was waived because this was a secondary analysis of de-identified data and this study was approved by the Institutional Review Board of the National Cancer Center, Korea (IRB number: NCC2020-0195). No administrative permission and/or licenses is acquired by this study to access the original data used in this research.

\section{Consent for publication}

Not applicable

\section{Conflict of Interest}

No potential conflict of interest relevant to this article was reported.

\section{Competing interests}

All authors declare no conflict of interest in this study.

\section{Author contributions}


Conceptualization: E-G Lee., Y-J Won., MC Lim., S-Y Jung.

Data curation: Y-J Won., JW Lim.

Formal analysis: Y-J Won., JW Lim.

Investigation: E-G Lee., Y-J Won., MC Lim., S-Y Jung., JW Lim

Methodology: Y-J Won., JW Lim.

Project administration: S-Y Jung

Software: JW Lim

Funding acquisition: Y-J Won., MC Lim., S-Y Jung.

Supervision: S-Y Jung, Y-J Won

Writing - original draft: E-G Lee.

Writing - review \& editing: E-G Lee., Y-J Won., MC Lim., S-Y Jung., HI Ha., YJ Chang.

\section{References}

1. Ferlay J, Soerjomataram I, Dikshit R, Eser S, Mathers C, Rebelo M, Parkin DM, Forman D, Bray FJljoc: Cancer incidence and mortality worldwide: sources, methods and major patterns in GLOBOCAN 2012. 2015, 136(5):E359-E386.

2. Jayson GC, Kohn EC, Kitchener HC, Ledermann JAJTL: Ovarian cancer. 2014, 384(9951):1376-1388.

3. Jung KW, Won YJ, Kong HJ, Lee ES: Cancer Statistics in Korea: Incidence, Mortality, Survival, and Prevalence in 2016. Cancer research and treatment : official journal of Korean Cancer Association2019, 51(2):417-430.

4. King M-C, Marks JH, Mandell JBJS: Breast and ovarian cancer risks due to inherited mutations in BRCA1 and BRCA2. 2003, 302(5645):643-646.

5. Domchek SM, Jhaveri K, Patil S, Stopfer JE, Hudis C, Powers J, Stadler Z, Goldstein L, Kauff N, Khasraw Met al: Risk of metachronous breast cancer after BRCA mutation-associated ovarian cancer. Cancer2013, 119(7):1344-1348.

6. Vencken PM, Kriege M, Hooning M, Menke-Pluymers MB, Heemskerk-Gerritsen BA, van Doorn LC, Collée MM, Jager A, van Montfort C, Burger CWet al: The risk of primary and contralateral breast cancer after ovarian cancer in BRCA1/BRCA2 mutation carriers: Implications for counseling. Cancer2013, 119(5):955-962.

7. Genetic/Familial High-Risk Assessment: Breast, Ovarian, and Pancreatic (Version 1.2020) [https://www.nccn.org/professionals/physician_gls/pdf/genetics_bop.pdf] 
8. Rebbeck TR, Kauff ND, Domchek SM: Meta-analysis of risk reduction estimates associated with riskreducing salpingo-oophorectomy in BRCA1 or BRCA2 mutation carriers. Journal of the National Cancer Institute2009, 101(2):80-87.

9. Bergfeldt K, Silfverswärd C, Einhorn S, Hall P: Overestimated risk of second primary malignancies in ovarian cancer patients. European journal of cancer (Oxford, England : 1990)2000, 36(1):100-105.

10. Levi F, Randimbison L, Blanc-Moya R, La Vecchia C: Second neoplasms after invasive and borderline ovarian cancer. European journal of cancer prevention : the official journal of the European Cancer Prevention Organisation (ECP)2009, 18(3):216-219.

11. Robinson E, Rennert G, Rennert HS, Neugut Al: Survival of first and second primary breast cancer. Cancer1993, 71(1):172-176.

12. Organization WH: International classification of diseases for oncology (ICD-0)-3rd edition, 1st revision. 2013.

13. Matsuo K, Mandelbaum RS, Machida H, Yoshihara K, Matsuzaki S, Klar M, Muggia FM, Roman LD, Wright JD: Temporal trends of subsequent breast cancer among women with ovarian cancer: a population-based study. Archives of gynecology and obstetrics2020, 301(5):1235-1245.

14. Lee EG, Kang HJ, Lim MC, Park B, Park SJ, Jung SY, Lee S, Kang HS, Park SY, Park Bet al: Different Patterns of Risk Reducing Decisions in Affected or Unaffected BRCA Pathogenic Variant Carriers. Cancer research and treatment : official journal of Korean Cancer Association2019, 51(1):280-288.

\section{Figures}


Figure 1.

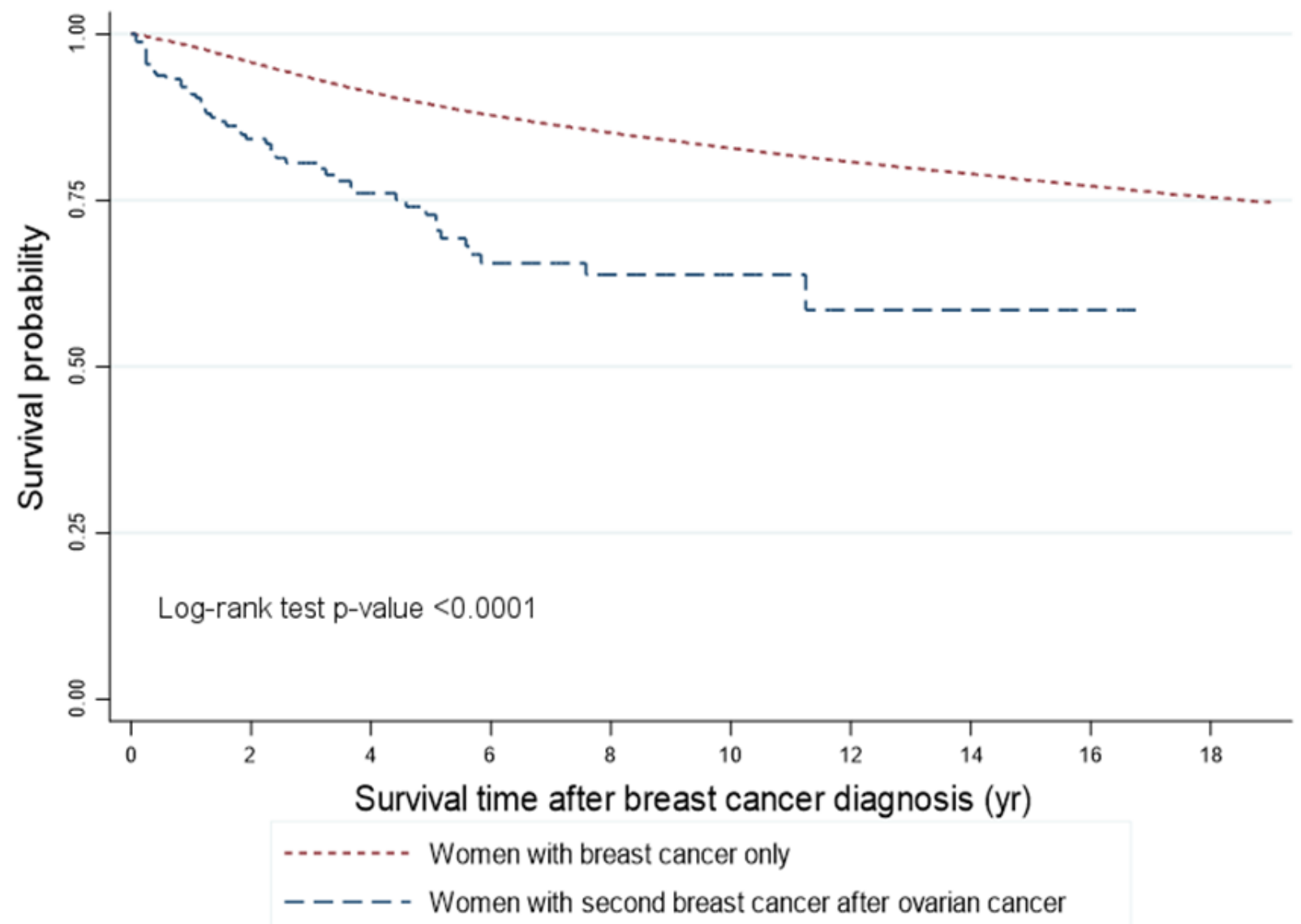

Figure 1

Survival outcomes of patients with breast cancer from the onset time of breast cancer diagnosis in Korea 
Figure 2.

A

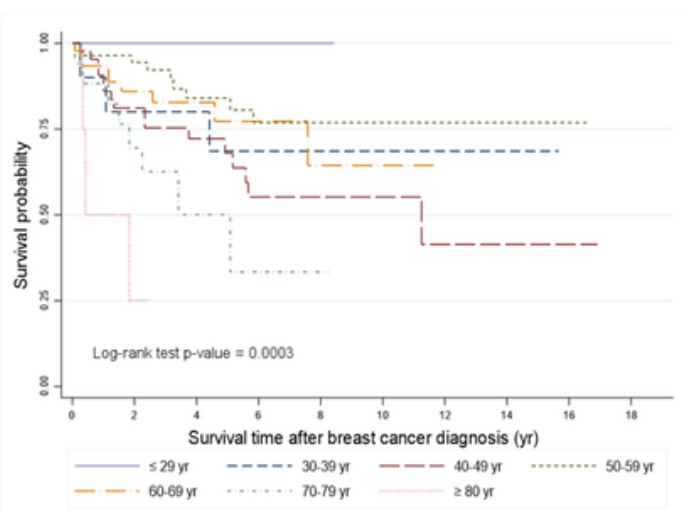

C

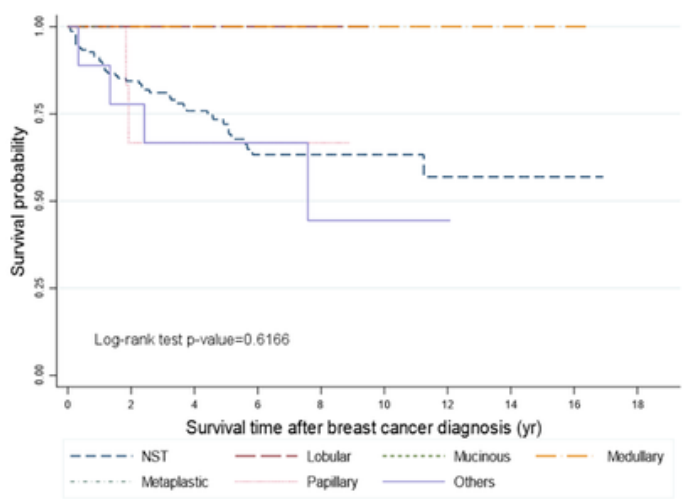

$E$

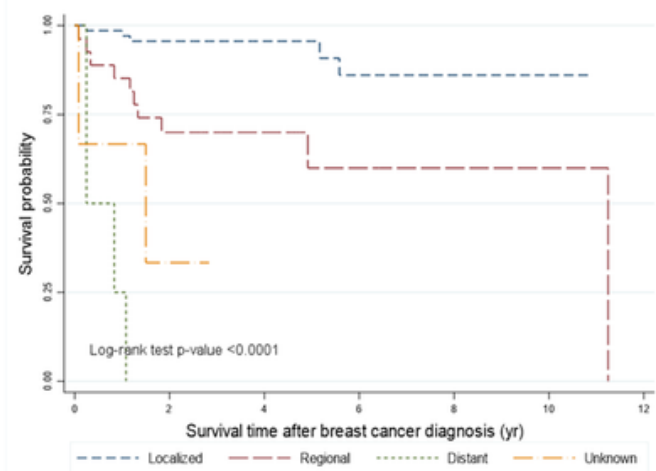

B

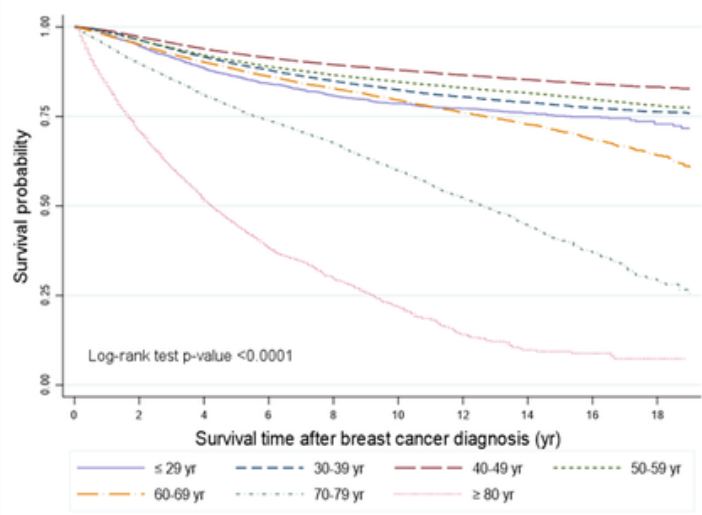

D

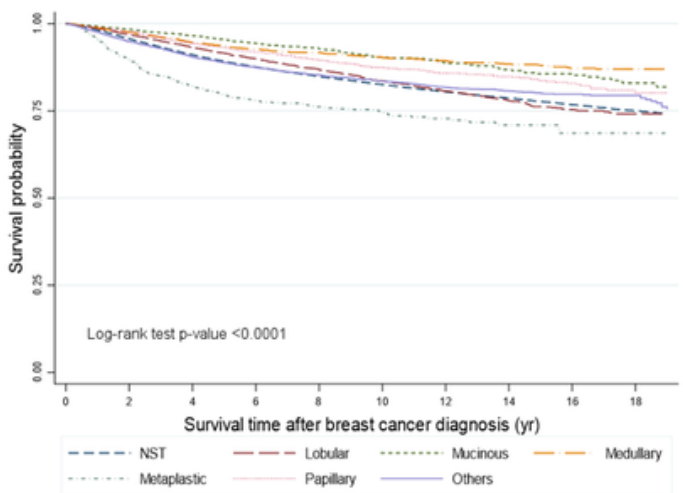

F

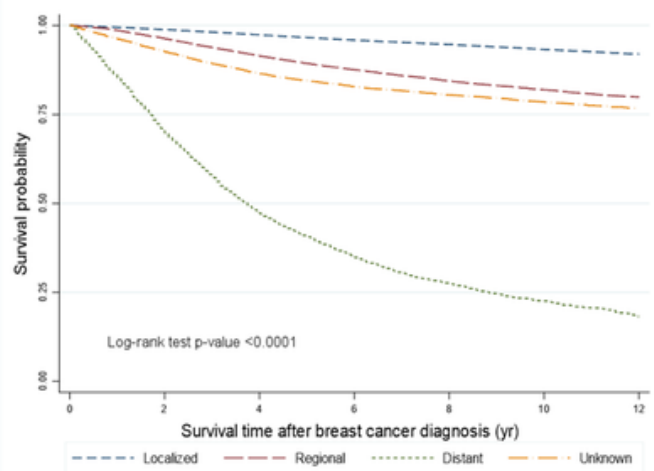

\section{Figure 2}

Survival outcomes from the onset time of breast cancer diagnosis according to clinicopathologic characteristics (A) Second primary breast cancer after ovarian cancer according to age groups (B) Primary breast cancer according to age groups (C) Second primary breast cancer after ovarian cancer according to histological types. (D) Primary breast cancer according to histological types. (E) Second 
primary breast cancer after ovarian cancer according to the SEER stage since 2006. (F) Primary breast cancer according to the SEER stage since 2006.

\section{Supplementary Files}

This is a list of supplementary files associated with this preprint. Click to download.

- SupplementaryTABLE1.docx 